\title{
Reduced Basis Approximations in Microwave Filters and Diplexers: Inf-Sup Constant Behavior
}

\author{
Sara García , Valentín de la Rubia and Michal Mrozowski
}

\begin{abstract}
In this work, a model order reduction technique for fast frequency sweep in microwave filters and diplexers via the Reduced Basis Method is detailed. The Finite Element Method is used to solve the time-harmonic Maxwell's equations in these resonant circuit problems. Taking into account the electromagnetic field varies smoothly as a function of the frequency parameter, a low dimension system parameter manifold is identified. Thus, the original frequency-dependent Finite Element problem can be approximated by a model of reduced size using a Reduced Basis approximation. Based on the field frequency behavior in these resonant structures, the Reduced Basis space where the approximation holds is identified and characterized. Finally, special emphasis is placed in the behavior of the infsup constant used to provide an a posteriori error estimator, and its consequences in the Reduced Basis approximation are highlighted.
\end{abstract}

Index Terms-Error analysis, finite element methods, Galerkin method, reduced basis methods, reduced order systems.

\section{INTRODUCTION}

Finite Element Methods (FEM) are specially suited for analysis of microwave filters and diplexers, where different dielectric materials as well as arbritrary geometry shapes need to be handled, either in the resonators themselves or in the final coupling layout required to reduce the size in the final device. However, its computational cost is a limiting factor. Model Order Reduction (MORe) techniques may be applied to speed up the computational efficiency. MORe consists of replacing a rather complex mathematical model by a much simpler approximated one that still maintains certain aspects of the original model [1].

Taken frequency as a parameter, the FEM problem we aim to solve turns into a frequency-dependent variational problem. This may be solved as independent FEM problems as many times as frequency points required in the frequency band of interest, resulting in a rather time-consuming approach. However, if we put together all these field solutions and determined how linearly independent these field solutions are, we would realize that all these field solutions live in a manifold of low dimension, induced by the parameter dependence. Finding a linear space of small dimension, namely, Reduced Basis space, approximating this manifold is the idea behind the Reduced Basis Method [2], [3], [4], [5]. As a result, the frequency-dependent variational problem can be solved in a fast frequency sweep MORe setting, applying Galerkin projection to the original FEM problem onto the Reduced Basis space.

By the same token, the reduced-order model needs to be certified and an a posteriori error analysis is developed. Different error estimators have been proposed [3], [4], [6], [7], [8] and some of them are based on the inf-sup constant in the frequency-dependent variational problem. However, whenever we hit a resonance in the frequency band of interest, which will be the case in as many times as the order of the microwave filter or diplexer, these inf-sup constant based error estimators are no longer valid. We take into account this problem and propose an altemative to certify the accuracy in the reducedorder model.

\section{PROBlEM STATEMENT}

The time-harmonic Maxwell's equations can be written in a classical variational problem over an appropriate admissible function space $\mathcal{H}$, viz.

$$
\begin{gathered}
\text { Find } E \in \mathcal{H} \text { such that } \\
a(E, v)=f(v) \quad \forall v \in \mathcal{H} .
\end{gathered}
$$

The bilinear form being

$$
a(E, v)=\int_{\Omega}\left(\frac{1}{\mu} \nabla \times E \cdot \nabla \times v-\omega^{2} \varepsilon E \cdot v\right) d V
$$

and the linear form $f(v)=j \omega \int_{\partial \Omega} J \cdot v d S=j \omega \int_{\Gamma} J \cdot v d S$. The frequency parameter, namely, angular frequency, is denoted as $\omega . \Omega \subset \mathbb{R}^{3}$ is a source-free bounded domain. The boundary $\partial \Omega$ of $\Omega$ is divided into perfect electric conductor (PEC) and ports, namely $\partial \Omega=\Gamma_{P E C} \cup \Gamma$. $E$ is the electric vector field, $\varepsilon$ and $\mu$ are, respectively, the permittivity and permeability of the medium, which is assumed to be lossless, and the tangential vector field $J$ is the current excitation at the ports. Here, the admissible space $\mathcal{H}$ stays in the Hilbert space $H($ curl,$\Omega)$ defined by:

$$
H(\operatorname{curl}, \Omega)=\left\{u \in L^{2}\left(\Omega, \mathbb{C}^{3}\right) \mid \nabla \times u \in L^{2}\left(\Omega, \mathbb{C}^{3}\right)\right\},
$$

equipped with the inner product

$$
(u, v)_{H(\mathrm{curl}, \Omega)}=(\nabla \times u, \nabla \times v)_{L^{2}(\Omega)}+(u, v)_{L^{2}(\Omega)},
$$


where

$$
\mathcal{H}=\left\{u \in H(\text { curl }, \Omega) \mid n \times u=0 \text { on } \Gamma_{P E C}\right\} .
$$

$n$ is the unit outward normal vector on the boundary $\partial \Omega$ of $\Omega$. Essential boundary conditions are considered in the solution space $\mathcal{H}$. Also, let us point out that the current excitation $J$ belongs to $H^{-\frac{1}{2}}($ div,$\partial \Omega)=\{n \times u \mid u \in H($ curl,$\Omega)\}$. We refer to [9], [10] for a throughout explanation of these spaces as well as their dual spaces.

Taking frequency as a parameter, we are interested in solving the following variational problem:

$$
\begin{gathered}
\text { Find } E(\omega) \in \mathcal{H} \text { such that } \\
a(E(\omega), v ; \omega)=f(v ; \omega) \quad \forall v \in \mathcal{H} .
\end{gathered}
$$

for every frequency $\omega$ in a given frequency band of interest $\left[\omega_{\min }, \omega_{\max }\right] \subset \mathbb{R}$. The well-posedness of problem (6) depends on the behavior of the so-called inf-sup constant $\beta$ as a function of frequency:

$$
\beta(\omega)=\inf _{u \in \mathcal{H}} \sup _{v \in \mathcal{H}} \frac{a(u, v ; \omega)}{\|u\|_{H(\text { curl }, \Omega)}\|v\|_{H(\text { curl }, \Omega)}} .
$$

Indeed, for all $\omega \in\left[\omega_{\min }, \omega_{\max }\right], \beta(\omega) \geq \beta_{0}$ and $\beta_{0}>0$ to ensure well-posedness and uniqueness in the variational problem (6), provided the bilinear form (2) is continuous.

In case we find an approximate solution to (6), $E_{N}$, we can bound the error in the approximation $\left\|E(\omega)-E_{N}\right\|_{H(\text { curl }, \Omega)}$ taking advantage of the inf-sup constant. Indeed, (7) can be rewritten as follows:

$$
\sup _{v \in \mathcal{H}} \frac{a(u, v ; \omega)}{\|u\|_{H(\text { curl }, \Omega)}\|v\|_{H(\text { curl }, \Omega)}}>\beta(\omega), \forall u \in \mathcal{H} .
$$

In particular, this inequality holds true taking $u \in \mathcal{H}$ as the field $E(\omega)-E_{N}$, which results in an upper bound for the error, namely,

$$
\left\|E(\omega)-E_{N}\right\|_{H(\operatorname{curl}, \Omega)}<\frac{1}{\beta(\omega)} \sup _{v \in \mathcal{H}} \frac{a\left(E(\omega)-E_{N}, v ; \omega\right)}{\|v\|_{H(\text { curl }, \Omega)}} .
$$

However, this error upper bound not only involves the computation of the norm in the residual functional $r\left(E_{N}, v ; \omega\right)=$ $a\left(E(\omega)-E_{N}, v ; \omega\right)=f(v ; \omega)-a\left(E_{N}, v ; \omega\right), \forall v \in \mathcal{H}$, i.e., $\left\|r\left(E_{N}, \cdot ; \omega\right)\right\|_{\mathcal{H}^{\prime}}=\sup _{v \in \mathcal{H}} \frac{r\left(E_{N}, v ; \omega\right)}{\|v\|_{H(c u r l, \Omega)}}$, which can be calculated in an efficient way, cf. [3], [5], but also the computation of the inf-sup constant for each frequency in the band. Determination of the inf-sup constant results in a computationally expensive task in the discrete setting, where a finite element approximation to the field solution is carried out to solve the variational problem (6) in an algebraic way. $\mathcal{H}_{h} \subset \mathcal{H}$ is a Nedelec finite element space approximating the infinite dimension space $\mathcal{H}$ and the discrete variational problem reads

Find $E(\omega) \in \mathcal{H}_{h}$ such that

$$
a(E(\omega), v ; \omega)=f(v ; \omega) \quad \forall v \in \mathcal{H}_{h} .
$$

Fig. 2 details the inf-sup constant behavior in a detuned $6^{t h}$ order combline filter as shown in Fig. 1. It should be pointed out that the inf-sup constant drops down to zero value at the resonance frequencies in the eletromagnetic structure, since microwave filters and diplexers are constructed by means of resonating modes. At these resonance frequencies, there is no uniqueness in the solution to Maxwell's equations, namely, the solution is an eigenmode, and this is why the inf-sup constant must drop down to zero. As a result, we can not rely on the inf-sup constant and the error upper bound in (9) to certify the accuracy in our approximation. A different strategy should be considered for this kind of microwave circuits.

\section{REDUCED BASIS APPROXIMATION}

Taking advantage of the smooth behavior of the field solution as a function of frenquency, we can solve the parameterdependent problem (10) in a more efficient way applying a Reduced Basis approximation. First, we need to identify a Reduced Basis space $\mathcal{W}^{N}$ capable of approximating the field solutions in (10) for all frequencies in the frequency band of interest, $\left[\omega_{\min }, \omega_{\max }\right]$, namely,

$$
\mathcal{M}_{h}^{\omega}=\left\{E(\omega) \in \mathcal{H}_{h}: \omega \in\left[\omega_{\min }, \omega_{\max }\right]\right\} \subset \mathcal{H}_{h} .
$$

Snapshots in this frequency band could be taken into account as a good starting point, i.e., $\mathcal{W}^{N}=\operatorname{span}\left\{E\left(\omega_{1}\right), \ldots, E\left(\omega_{N}\right)\right\}$, where $\omega_{1}, \ldots, \omega_{N}$ $\in\left[\omega_{\min }, \omega_{\max }\right]$. The Reduced Basis approximation to problem (10) then reads

$$
\begin{gathered}
\text { Find } E(\omega) \in \mathcal{W}^{N} \text { such that } \\
a(E(\omega), v ; \omega)=f(v ; \omega) \quad \forall v \in \mathcal{W}^{N} .
\end{gathered}
$$

This allows us to find an approximated solution to (10) by solving a problem of reduced size, $N$, which gives rise to our reduced-order model. The question that still remains to be answered is how to optimally select the snapshots in the Reduced Basis space. $\mathcal{W}^{N} \subset \mathcal{M}_{h}^{\omega}$ and the Reduced Basis space $\mathcal{W}^{N}$ should be constructed in such a way that, with the minimum number of basis vectors, spans a space that is close enough to $\mathcal{M}_{h}^{\omega}$. In order to do so, snapshots in $\mathcal{W}^{N}$ should be as linearly independent as possible, and ideally, they should be chosen to be orthogonal solutions among the available field solutions in the parameter-induced manifold $\mathcal{M}_{h}^{\omega}$. Since we are addressing resonanting mode structures, such as microwave filters and diplexers, eigenmodes resonanting in the frequency band of interest are present in the manifold $\mathcal{M}_{h}^{\omega}$ and constitute an orthogonal set of solutions. These eigenmodes are therefore a natural choice to set the Reduced Basis space up. However, this eigenmode space itself is not enough to solve for the driven problem (10) and this space has to be enlarged with additional snapshots in the frequency band of interest. For the selection of these additional snapshots, a greedy strategy based on the residual evalution is carried out [3], [5].

\section{NUMERICAL RESULTS}

In this section, we apply the Reduced Basis approximation strategy described earlier to a detuned $6^{\text {th }}$ order combline filter. The FEM analysis is carried out using InventSIM framework [11]. Fig. 3 shows the $S$ parameter filter response 


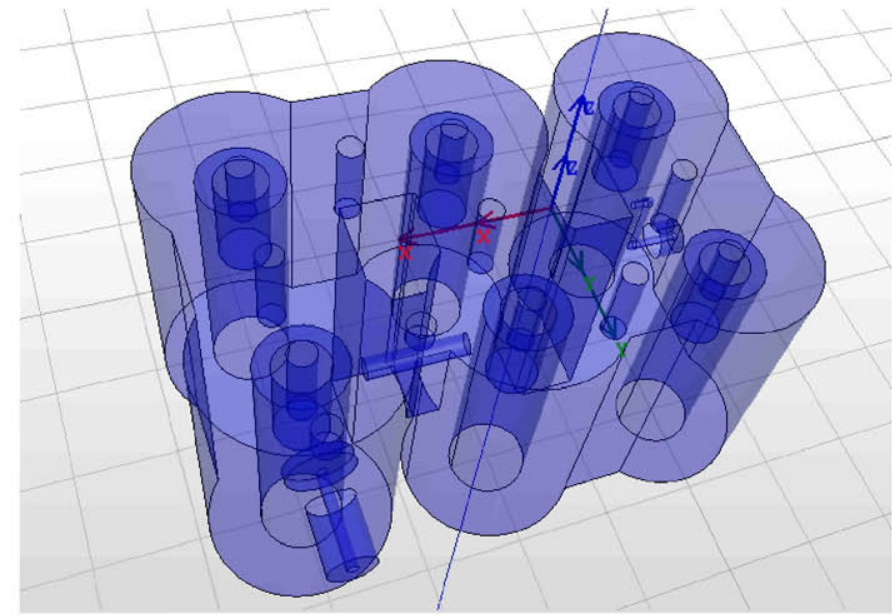

Fig. 1. $6^{\text {th }}$ order combline filter.

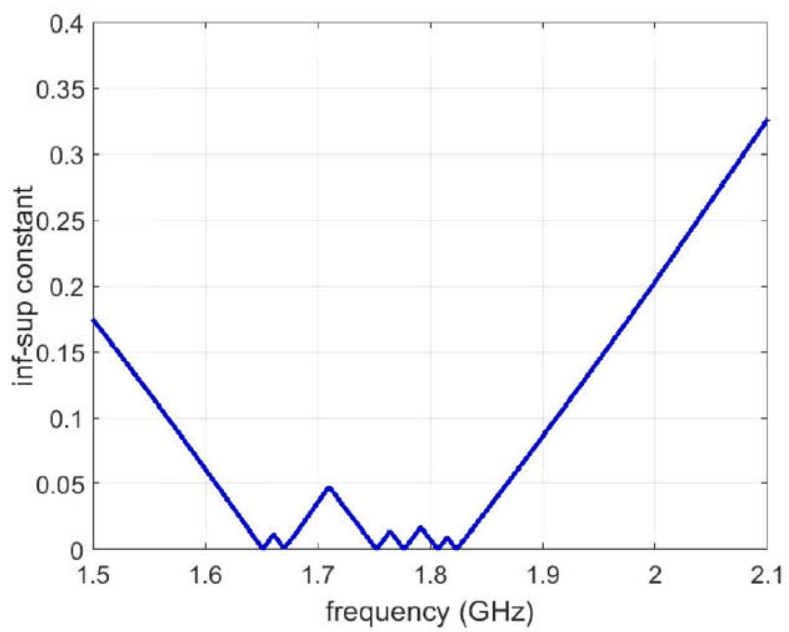

Fig. 2. Inf-sup constant behavior in the $6^{t h}$ order combline filter.

as we increase the dimension of the Reduced Basis space $\mathcal{W}^{N}$. The first basis vectors taken into account in the Reduced Basis approximation are the 6 eigenmodes hit in the frequency band of interest. It should be noted that it is not until we have taken into account these 6 eigenmodes for the $6^{\text {th }}$ order combline filter when we can achieve extremely fast convergence to the driven problem after adding a few additional snapshots in the frequency band of interest.

\section{CONCLuSion}

A Reduced Basis approximation for fast frequency sweep in resonanting microwave circuits has been proposed. An appropriate Reduced Basis space for MORe analysis has been detailed and special emphasis has been placed in the behavior of the inf-sup constant for this kind of resonant circuits. The a posteriori error analysis based in the inf-sup constant has been shown to be no longer available for this kind of microwave circuits.

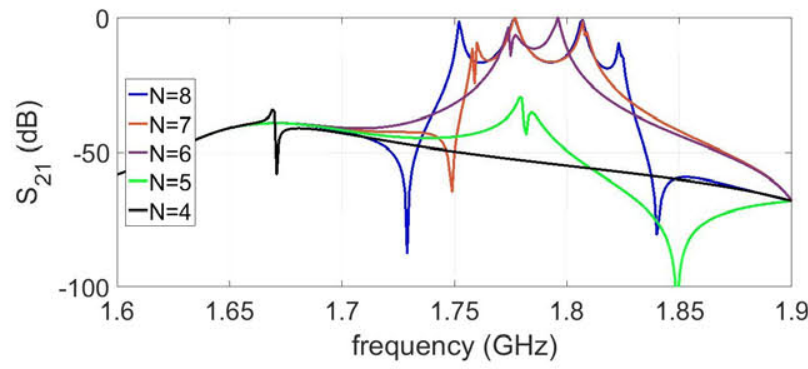

Fig. 3. Reduced Basis approximation of the $\mathrm{S}$ parameter response in the detuned $6^{t h}$ order combline filter as the number of basis vectors $N$ in the Reduced Basis space changes.

\section{ACKNOWLEDGEMENT}

This work was partially supported by the TEAM-TECH project entitled EDISOn - Electromagnetic Design of flexIble SensOrs operated within the Foundation for Polish Science TEAM TECH Programme co-financed by the European Regional Development Fund, Operational Programme Smart Growth 2014-2020, and also by the Polish National Science Centre under Contract UMO-2012/07/B/ST7/01241.

\section{REFERENCES}

[1] A. C. Antoulas, Approximation of Large-Scale Dynamical Systems. Philadelphia, PA: SIAM, 2005.

[2] A. Quarteroni, A. Manzoni, and F. Negri, Reduced Basis Methods for Partial Differential Equations. Springer, 2016.

[3] V. de la Rubia, U. Razafison, and Y. Maday, "Reliable fast frequency sweep for microwave devices via the reduced-basis method," IEEE Trans. Microwave Theory Tech., vol. 57, no. 12, pp. 2923-2937, Dec. 2009.

[4] M. Rewienski, A. Lamecki, and M. Mrozowski, "A goal-oriented error estimator for reduced basis method modeling of microwave devices," IEEE Microwave Wireless Compon. Lett., vol. 25, no. 4, pp. 208-210, Apr. 2015.

[5] A. Sommer, O. Farle, and R. Dyczij-Edlinger, "A new method for accurate and efficient residual computation in adaptive model-order reduction," IEEE Trans. Magn., vol. 51, no. 3, pp. 1-4, Mar. 2015.

[6] W. Wang, G. N. Paraschos, and M. N. Vouvakis, "Fast frequency sweep of fem models via the balanced truncation proper orthogonal decomposition,” IEEE Trans. Antennas Propagat., vol. 59, no. 11, pp. 4142-4154, Nov. 2011.

[7] M. W. Hess and P. Benner, "Fast evaluation of time-harmonic Maxwell's equations using the reduced basis method," IEEE Trans. Microwave Theory Tech., vol. 61, no. 6, pp. 2265-2274, Jun. 2013.

[8] M. W. Hess, S. Grundel, and P. Benner, "Estimating the inf-sup constant in reduced basis methods for time-harmonic Maxwell's equations," IEEE Trans. Microwave Theory Tech., vol. 63, no. 11, pp. 3549-3557, Nov. 2015.

[9] V. Girault and P.-A. Raviart, Finite Element Methods for Navier-Stokes Equations. Berlin: Springer-Verlag, 1986.

[10] P. Monk, Finite Element Methods for Maxwell's Equations. New York, NY: Oxford Univ. Press, 2003.

[11] A. Lamecki, L. Balewski, and M. Mrozowski, "An efficient framework for fast computer aided design of microwave circuits based on the higherorder 3D finite-element method," Radioengineering, vol. 23, no. 4, p 971, 2014. 